Research Article

\title{
Integrated Analysis of Long Noncoding RNA Expression Profiles in Acute-on-Chronic Liver Failure
}

\author{
Xiaoyu Fu, ${ }^{1}$ Da Cheng, ${ }^{1}$ Yi Ouyang, ${ }^{1}$ Ying Li, ${ }^{1}$ Ronghua $\mathrm{Li}^{2}$ Shifang Peng $\mathbb{D}^{1},{ }^{1}$ and Lei Fu $\mathbb{D}^{1}$ \\ ${ }^{1}$ Department of Infectious Diseases, Hunan Key Laboratory of Viral Hepatitis, Xiangya Hospital, Central South University, \\ Changsha, 410008 Hunan, China \\ ${ }^{2}$ Department of Nuclear Medicine, Xiangya Hospital, Central South University, Changsha, 410008 Hunan, China
}

Correspondence should be addressed to Shifang Peng; psfang_xy@sohu.com and Lei Fu; fufu.lei@outlook.com

Received 30 June 2020; Revised 10 March 2021; Accepted 26 April 2021; Published 19 May 2021

Academic Editor: Naohiko Masaki

Copyright (c) 2021 Xiaoyu Fu et al. This is an open access article distributed under the Creative Commons Attribution License, which permits unrestricted use, distribution, and reproduction in any medium, provided the original work is properly cited.

People infected with chronic hepatitis B virus (HBV) might progress to acute-on-chronic liver failure (ACLF) with a high fatality rate. Long noncoding RNAs (lncRNAs) are involved in human diseases, but it is unknown whether lncRNAs are involved in the progression of chronic HBV infection to ACLF. Hence, this study is aimed at systemically identifying and characterizing the landscape and the molecular mechanism of lncRNAs in the pathogenesis of chronic HBV infection progress to ACLF. RNA sequencing (RNA-Seq) of peripheral blood samples from $5 \mathrm{ACLF}$ and $5 \mathrm{HBV}$ infection patients was performed. We detected 9733 lncRNAs, including 406 annotated lncRNAs and 9327 novel lncRNAs. A total of $407 \operatorname{lncRNAs}$ were found to be significantly dysregulated in the patients with ACLF as compared with those in the chronic HBV infection patients. The flanking protein-coding genes of differentially expressed lncRNAs were enriched with pathways that might contribute to the pathogenesis of ACLF, such as the WNT signaling pathway. Furthermore, 9 selected differentially expressed lncRNAs validated by the qRT-PCR, showing that the expression patterns of these 9 lncRNAs were consistent with the RNA-Seq data. Four selected differentially expressed lncRNAs were also validated in another patient cohort comprising 80 patients with ACLF and 65 patients with chronic HBV infection. Aberrant lncRNAs might be used to develop novel diagnostic biomarkers or drug targets for ACLF.

\section{Introduction}

Hepatitis B virus (HBV) is a global epidemic disease. According to a report from the World Health Organization, approximately 2 billion people worldwide are infected with HBV, with 240 million of them presenting chronic HBV infections. Furthermore, approximately 650000 people died from liver failure, cirrhosis, and hepatocellular carcinoma (HCC) induced by HBV infection [1]. In China, 93 million people are infected with chronic HBV every year, and approximately $30 \%$ patients present spontaneous hepatitis acute exacerbation, with some patients progressing to acute-on-chronic liver failure (ACLF) with a high fatality rate. ACLF can lead to severe clinical syndromes such as jaundice, blood coagulation dysfunction, hepatic encephalopathy, and ascites [2,3]. In recent years, experts have considered ACLF as a new type of liver disease with short-term mortality rate (generally 28 days) and dysfunctional systematic inflammatory response. It is difficult to distinguish the initial clinical symptoms between ACLF and severe chronic hepatitis. Moreover, the short-term case fatality rate reaches up to $>50 \%$ when the disease progresses to liver failure [4]. Although liver transplantation is the only effective treatment approach for liver failure, most patients cannot benefit from liver transplantation owing to source limitation of donor liver, economic pressure, and operation risks [2]. Therefore, it is important to prevent the occurrence of liver failure during the early clinical period.

In China, the general cause of ACLF is hepatitis virus, mainly HBV (approximately 80-85\%). Patients with chronic HBV have a long progression window from acute exacerbation of hepatitis to ACLF, which is known as "acute on- 
chronic pre-liver failure" (pre-ACLF) [3]. The initial clinical manifestations are highly similar between chronic HBV infection and ACLF. With the dynamic development of the disease, some patients might recover gradually from severe chronic hepatitis, without liver failure, whereas others might develop to ACLF due to further aggravation of liver failure. The pathogenesis of ACLF is extremely complex and is affected by various factors, but the specific mechanism is not known. Thus, it is important to discover molecular mechanisms underlying the progression of chronic HBV infection to ACLF, identify clinical biomarkers to predict or diagnose in time, and assist in the development of necessary intervention and treatment for reducing the risk of death in patients with ACLF.

A large fraction of human transcripts is long noncoding RNAs (lncRNAs), as only approximately $1 \%$ of human transcripts encode proteins $[5,6]$. The lncRNAs are spliced, polyadenylated transcripts, of size $>200 \mathrm{bp}$, and they lack the potential to encode proteins [7-9]. IncRNAs play important roles in several important biological processes, such as cell proliferation, differentiation, and apoptosis [6, 10-13]. A common example is the inactivation of $\mathrm{X}$ chromosome through cis-acting XIST lncRNA [14]. Recent studies have showed that dysfunctional lncRNAs might be involved in the development of liver diseases, such as liver fibrosis [15, 16]. For example, Song et al. have reported that the activation of hepatic lncRNA H19 could promote cholestatic liver fibrosis through the ZEB1-EpCAM signaling pathway in mice [15]. In addition, several lncRNAs have been shown to be dysregulated in HCC tissues, and they can act as potential biomarkers for diagnosing HCC and predicting the prognosis and response to therapy $[17,18]$. For instance, lncRNA HULC is specifically expressed in hepatocytes and highly upregulated in liver cancer. It plays an important role in tumorigenesis and is associated with the intrahepatic metastases, HCC recurrence, and postoperative survival $[19,20]$. Despite these findings of a few IncRNAs linked with liver diseases, it is not clear whether IncRNAs are involved in the pathogenesis of ACLF.

RNA sequencing (RNA-Seq) has been widely used to study whole transcriptome changes under different conditions, in order to quantify gene expression with dynamic range and overcome the shortcomings of the microarray technology [21, 22]. Recent advances in RNA-Seq and computational methods for reconstructing transcriptome offer an excellent opportunity to annotate and characterize IncRNAs, and a large number of lncRNAs have been identified using RNA-Seq. For example, Cabili et al. have identified more than 8000 human long intergenic noncoding RNAs using RNA-Seq data and showed that most of them have not been previously described [13]. Therefore, abundant RNA-Seq data could enable to comprehensively identify and quantify lncRNAs (also protein-coding genes) under pathological conditions.

In this study, to explore the biological role of lncRNAs in the pathogenesis mechanism in the progression of chronic HBV infection to ACLF, we performed RNA-Sequencing of samples from 5 patients with ACLF and 5 patients with HBV infection (chronic asymptomatic HBV carrier, AsC).
By analyzing the RNA-Seq data, we identified aberrantly expressed lncRNAs and protein-coding genes between ACLF and AsC patients. Moreover, the Gene Ontology (GO) and Kyoto Encyclopedia of Genes and Genomes (KEGG) pathway analyses were performed to predict the biological roles and potential signaling pathways of these differentially expressed lncRNAs. In addition, an IncRNA-mRNA network analysis was conducted to further explore the potential roles of differentially expressed lncRNAs in ACLF pathogenesis. Furthermore, we performed quantitative real-time PCR (qRT-PCR) analysis to confirm that the expression of 15 lncRNAs in another larger cohort comprising patients with ACLF due to HBV infection. The dysfunctional expression of lncRNAs might serve as potential biomarkers of ACLF, and aberrant lncRNAs involved in ACLF might open new avenues for the diagnosis and treatment of liver failure in human in the future.

\section{Materials and Methods}

2.1. Patients and Samples. From January 2016 to May 2017, 5 patients with ACLF and 5 patients with HBV (chronic asymptomatic HBV carrier, AsC) from the Xiangya Hospital of Centre-South University (Hunan, China) were enrolled in the present study. Blood samples were collected from all patients. These patients were all infected with HBV. Patients with liver damage caused by drinking, drugs, and other factors were excluded. 5 ACLF patients were diagnosed according to APSAL diagnostic criteria. Among them, 2 patients had not received nucleoside analogues (NAs) treatment, and $\mathrm{HBV}$ was in an active replication state in these 2 patients. 3 patients had received NA treatment, and the drugs were discontinued irregularly for more than half a year. All patients were treated with NAs after being diagnosed with ACLF. There were no patients with hepatic encephalopathy, and all these 5 ACLF patients were in the early stage of ACLF. The prognosis of 5 ACLF patients is improved and discharged. AsC exhibited HBsAg positive for more than 6 months, HBV DNA positive, and $\mathrm{HBeAg}$ negative, or positive. However, serum ALT and AST continue to be at normal levels. These patients were followed up 3 times in 1 year with an interval of more than 3 months. Patients with $\mathrm{AsC}$ were diagnosed and excluded as previous reported [23, 24]. The clinical characteristics of these 10 patients are summarized in Table 1 and Supplementary Table 2. We used these samples to perform RNA-Seq.

Another cohort, comprising 80 patients with ACLF and 65 patients with HBV infection were recruited from the Xiangya Hospital of Centre-South University (Hunan, China). These samples were used to verify the expression of differentially expressed lncRNAs through qRT-PCR. The clinical characteristics of these 145 patients are shown in Supplementary Table 3.

All individuals provided a written informed consent for use of their samples in this study. The present study was approved by the Ethics Committee of the Xiangya Hospital of Centre-South University (2017-P2-084-01). 
TABLe 1: Clinical characteristics of 10 patients.

\begin{tabular}{|c|c|c|c|c|c|c|c|c|}
\hline Groups & Gender & Age & $\operatorname{ALB}(g / L)$ & TBIL $(\mu \mathrm{mol} / \mathrm{L})$ & DBIL $(\mu \mathrm{mol} / \mathrm{L})$ & $\mathrm{TBA}(\mu \mathrm{mol} / \mathrm{L})$ & ALT (U/L) & AST (U/L) \\
\hline \multirow{5}{*}{ AsC group } & $\mathrm{F}$ & 28 & 48.2 & 11.6 & 6.4 & 8.2 & 33.5 & 19 \\
\hline & $\mathrm{F}$ & 31 & 50.3 & 12.2 & 5.8 & 10.1 & 29.7 & 20.8 \\
\hline & M & 27 & 53.8 & 9.1 & 3.9 & 1.7 & 32.8 & 23.6 \\
\hline & M & 33 & 47.7 & 10.5 & 4.9 & 5 & 20.5 & 21.2 \\
\hline & M & 39 & 46.9 & 9.8 & 5.3 & 3.9 & 19.7 & 17.3 \\
\hline \multirow{5}{*}{ ACLF group } & M & 36 & 21.7 & 307.3 & 178.5 & 101.4 & 409.3 & 189.7 \\
\hline & M & 52 & 35.8 & 618.1 & 321.7 & 137.3 & 322.5 & 237.3 \\
\hline & $\mathrm{F}$ & 50 & 34 & 508.8 & 260.4 & 189.8 & 707.1 & 433.4 \\
\hline & $\mathrm{F}$ & 45 & 31.1 & 376.4 & 147.5 & 126.9 & 609.4 & 176.1 \\
\hline & M & 63 & 29.6 & 679.6 & 319.3 & 132.3 & 1033.5 & 770.2 \\
\hline
\end{tabular}

2.2. RNA-Seq Library Preparation and Sequencing. The peripheral blood was obtained from the patients on the day of admission, and these patients had already suffered from ACLF at the time of admission. Next, the generation sequencing of RNA was performed using peripheral blood samples obtained from the patients. The total RNA was extracted from peripheral blood samples using Trizol reagent (Invitrogen, CA, USA), following the manufacturer's instruction. The RNA quantity and purity were analyzed using a Bioanalyzer 2100 and RNA 6000 Nano LabChip Kit (Agilent, CA, USA) with the RIN (RNA Integrity Number) $>7.0$. Approximately $10 \mu \mathrm{g}$ of total RNA representing a specific adipose type was used to deplete the ribosomal RNA according to the instructions of the Epicentre Ribo-Zero Gold Kit (Illumina, San Diego, USA). Following purification, the poly (A) - or poly (A) + RNA fractions were fragmented into small pieces using divalent cations under elevated temperature. The cleaved RNA fragments were then reversetranscribed to create the final cDNA library in accordance with the protocol of the mRNA-Seq Sample Preparation Kit (Illumina, San Diego, USA); the average insert size for the paired-end libraries was $300 \mathrm{bp}$ ( $\pm 50 \mathrm{bp}$ ). We also performed paired-end sequencing on Illumina X10 (lc-bio, China), following the vendor's recommended protocol.

2.3. Human Gene Annotations. Human reference genome sequence of GRCh38 version was collected from the ENSEMBL database. Human gene annotations of proteincoding genes and lncRNAs of GRCh38 version were also downloaded from the ENSEMBL database.

2.4. Bioinformatics Analysis of the Transcriptome Data. For the raw RNA-Seq data, we firstly assessed the quality of sequencing reads using the Fastqc software (v 0.10.1) and then discarded low quality reads that showed quality score less than 10 , contained the rates of base $\mathrm{N}$ more than $5 \%$, or were contaminated by adaptors, as well as host sequences with $90 \%$ sequence similarity using Cutadpter (v 1.10) tool. Next, we aligned the cleaned reads of each sample to the GRCh38 reference genome through the TopHat software ( $\mathrm{v}$ 2.0.4) [25], a gapped aligner capable of discovering splice junctions. The aligned reads from TopHat were then assembled into transcriptome, and the gene expression was quanti- fied using StringTie (v 1.3.0) tool [26], which de novo assembles and quantitates full-length transcripts representing multiple splice variants for each gene locus.

2.5. Identification of Known and Prediction of Novel lncRNAs. For the identification of known and novel lncRNAs involved in ACLF, a bioinformatics computational pipeline was developed (Supplementary Figure 1). Firstly, all the transcripts assembled using StringTie were annotated with gene annotations of the GRCh38 protein-coding genes and lncRNAs, if the transcripts overlapped with the proteincoding genes, then the transcripts were considered as protein-coding genes. If the remaining transcripts overlapped with IncRNAs, then the transcripts were considered as known lncRNAs. The remaining unannotated transcripts were used for identifying potential novel lncRNAs. Secondly, these unannotated transcripts were filtered if the transcript length was less than $200 \mathrm{bp}$, the exon number was less than 2, and the coverage of reads number was less than 3 . Finally, the remaining transcripts were evaluated for the coding potential using both Coding Potential Calculator (CPC) [27] and Coding-Non-Coding Index (CNCI) [28]. The transcripts with a CPC score $<-1$ and $\mathrm{CNCI}$ score $<0$ were considered as potential novel lncRNAs.

2.6. Identification of Differentially Expressed Protein-Coding Genes and $\operatorname{lncRNAs}$ between the ACLF and AsC Groups. Ballgown algorithm was used to compare the ACLF and AsC groups to identify differentially expressed protein-coding genes and lncRNAs. The protein-coding genes and lncRNAs with $\geq 2$-fold change in expression and statistical significance $(p<0.05)$ were considered as differentially expressed.

2.7. Functional Enrichment Analysis. The significance of enriched GO terms and KEGG pathways for the proteincoding genes was determined using the GOseq software (v1.18.0) and KOBAS (v2.0) [29]. The false discovery rate (FDR; corrected $p$ value) of $<0.05$ was set as the cutoff for significant GO terms and KEGG pathways.

2.8. The $q R T-P C R$ Validation of $\operatorname{lncRNAs}$. We selected top 15 differentially expressed lncRNAs to perform qRT-PCR to validate their expression in the 10 patients. In addition, nine 
lncRNAs were validated in another cohort, comprising 80 patients with ACLF and 65 patients with HBV infection. The total RNA was reversed transcribed into cDNA using SuperScript III Reverse Transcriptase (Invitrogen, Grand Island, NY, USA) in accordance with the manufacturer's instructions. An Applied Biosystems ViiA 7 Real-Time PCR System and $2 \times$ PCR Master Mix were used to perform the qRT-PCR (Arraystar). The reaction conditions were as follows: incubation at $95^{\circ} \mathrm{C}$ for $10 \mathrm{~min}$, followed by 40 cycles of $95^{\circ} \mathrm{C}$ for $10 \mathrm{~s}$ and $60^{\circ} \mathrm{C}$ for $1 \mathrm{~min}$. The relative lncRNA expression was calculated using the $2^{-\Delta \Delta \mathrm{Ct}}$ method and was normalized to that of GAPDH, as an endogenous reference transcript [30]. The specific primers for each lncRNAs are listed in Table 2.

2.9. Target Gene Prediction and Functional Analysis of lncRNAs. IncRNAs might play a cis-acting element role on the neighboring target genes. To explore the function of lncRNAs, we predicted the cis-target genes of lncRNAs. In this study, the coding genes 100000 bp upstream and downstream were collected. The functional analysis of the target genes for lncRNAs was then performed using BLAST2GO [31]. A function was considered significantly enriched if the $p$ value was $<0.05$ by using a hypergeometric test.

\section{Results}

3.1. Expression Landscape of $\operatorname{lncRNAs}$ during the Progression of $A s C$ to $A C L F$. ACLF is a serious threat to human health. Therefore, we aimed to explore the pathogenesis mechanism of ACLF and characterize a comprehensive expression landscape of lncRNAs during the progression of AsC to ACLF. By aligning the sequenced reads to human reference sequences, we found that on an average 95 million unique reads per samples were mapped to human reference sequence (Supplementary Table 1). To depict the landscape of lncRNAs during the progression of AsC to ACLF, we performed a bioinformatics analysis using the transcriptome data. First, we used StringTie tool to assemble the aligned reads to transcripts. The assembled transcripts were then annotated with annotations of the GRCh38 protein-coding genes and lncRNAs to separate the protein-coding genes and known lncRNAs. If the transcripts were not overlapped with the protein-coding genes but overlapped with the annotated IncRNAs, then the transcripts were considered as known lncRNAs. To characterize a comprehensive profile of lncRNAs, the remaining unannotated transcripts were used for identifying potential novel lncRNAs (Supplementary Figure 1). Briefly, the unannotated transcripts were discarded by length $(<200 \mathrm{bp})$, the exon number $(<2)$, and the coverage of reads number $(<3)$. Finally, the remaining transcripts were predicted for the coding potential using both CPC and CNCI algorithm. The transcripts with a CPC score $<-1$ and CNCI score $<0$ were considered as potential novel lncRNAs.

We identified 9733 lncRNAs during the progression of AsC to ACLF, including 406 known lncRNAs and 9327 novel lncRNAs. Moreover, some well-known lncRNAs were expressed during the progression of AsC to ACLF, such as
lncRNA GAS5, MALAT1, and NEAT1. To depict the features of lncRNAs, we compared the lncRNAs and proteincoding genes (Supplementary Figure 2). The number of expressed lncRNAs was less than that of the protein-coding genes (Supplementary Figure 2A). The expression level of IncRNAs was comparable with that of the protein-coding genes. In addition, the exon number and length of the protein-coding genes were more than those of the lncRNAs (Supplementary Figure 2B, C). However, the predicted open reading frame (ORF) length of lncRNAs was less than that of the protein-coding genes (Supplementary Figure 2D, lncRNAs mean ORF: 83; protein-coding genes mean ORF: 316), which is consistent with the findings of previous studies that lncRNAs have relatively lower coding potential $[10,13]$. Overall, these results suggested that lncRNAs play an important role in the transcriptional landscape of the hepatic gene expression and the dysfunctional expression of lncRNAs might be involved in the progression of $\mathrm{AsC}$ to ACLF.

3.2. Differentially Expressed IncRNAs in the Progression of AsC to ACLF. To identify lncRNAs differentially expressed in the ACLF group as compared with that in the AsC group, we performed a differential expression analysis using Ballgown algorithm, which is considered a robust method to analyze the RNA-Seq data [32]. We found that 768 differentially expressed protein-coding genes with $\geq 2$ fold change between the ACLF and the AsC groups (FDR < 0.001), including 483 upregulated and 285 downregulated protein-coding genes (Figures 1(a) and 1(b)). For lncRNAs, we obtained 407 differentially expressed lncRNAs in the ACLF group by comparing with those of the AsC group, including 155 upregulated and 252 downregulated lncRNAs (Figure 1(c)). Based on the differentially expressed lncRNAs, we could clearly divide the ACLF and AsC groups (Figure 1(d)). For example, the IncRNA THRB showed a 3.2-fold upregulation in the ACLF group compared with that in the AsC group. In summary, the aberrant expression of lncRNAs might be involved in the pathogenesis of the progression of AsC to ACLF.

3.3. Validation of Differentially Expressed IncRNAs Using $q R T-P C R$. To demonstrate the expression of lncRNAs, we selected the top 15 differentially expressed lncRNAs (Table 2) with a variable fold change to validate by qRTPCR. The results revealed that the expression of nine IncRNAs was consistent with the RNA-Seq data (Figure 2(a), Supplementary Figure 3A). Furthermore, we compiled a larger patient cohort comprising 80 patients with ACLF and 65 patients with HBV to perform the qRTPCR experiment to validate the expression of the nine selected differentially expressed lncRNAs. The results were consistent with the change in gene expression observed in the previous cohort. As shown in Figure 2(b), we also found that four lncRNAs (RP11-25K21.6, THRB, RAB27A, and GNPTAB) out of nine lncRNAs (Supplementary Figure 3B) were significantly differentially expressed between the ACLF and the AsC groups. The lncRNAs RP11-25K21.6 and THRB were downregulated in the ACLF group compared with those in the AsC group. Whereas, the 
TABLE 2: lncRNA primer sequences for qRT-PCR.

\begin{tabular}{|c|c|c|}
\hline Number & $\operatorname{lncRNA}$ & Sequence $\left(5^{\prime}\right.$ to $\left.3^{\prime}\right)$ \\
\hline \multirow{2}{*}{1} & lncRNA RP11-25K21.6-F & GCGTTGGTGGTATAGTGGTGAGC \\
\hline & lncRNA RP11-25K21.6-R & ACACAGCGCGTTATAGGTTCTGAC \\
\hline \multirow{2}{*}{2} & lncRNA THRB-F & GCACTTGAGACACTCTGGTCGTTC \\
\hline & lncRNA THRB-R & GCCACATCTCATCCAGACCACTTG \\
\hline \multirow{2}{*}{3} & lncRNA PLCB1-F & ACCAGTGGGATGGTAGAAGGT \\
\hline & lncRNA PLCB1-R & TCTTTGCTAGCTGTTTAGCACG \\
\hline \multirow{2}{*}{4} & lncRNA TRIO-F & CTGCCAGTTGTTCTTTTGCAGG \\
\hline & lncRNA TRIO-R & GGGAAAGTAAGGGACTCGGGA \\
\hline \multirow{2}{*}{5} & lncRNA DOK6-F & TGTAGCCTGAGCATCCCCTT \\
\hline & lncRNA DOK6-R & ATCCTGGAGGGCTTGGATGG \\
\hline \multirow{2}{*}{6} & lncRNA APTX-F & GTTTATGGGACAGGTGATACCTCA \\
\hline & lncRNA APTX-R & ACAGGGTTGCTAAGATGATGAAATG \\
\hline \multirow{2}{*}{7} & lncRNA ST3GAL4-F & CAGATCCCAGCTCAAAGGCG \\
\hline & lncRNA ST3GAL4-R & TTCGGGAGAGCACTCAGAGG \\
\hline \multirow{2}{*}{8} & lncRNA MSTRG.7502-F & CTTGCTCATCGATTCCAATGATTCC \\
\hline & lncRNA MSTRG.7502-R & CTTTCCAAGTTTTGCCCCCTG \\
\hline \multirow{2}{*}{9} & lncRNA ZBTB16-F & GGAGCTCAACAGGCACACAA \\
\hline & lncRNA ZBTB16-R & AGCAGCAGCATTGTGACTCC \\
\hline \multirow{2}{*}{10} & lncRNA MSTRG.90039-F & TCATGGGGTCGGTACCAAGG \\
\hline & lncRNA MSTRG.90039-R & CATCTGCACTGGAGGAGGGA \\
\hline \multirow{2}{*}{11} & lncRNA PAPSS1-F & TCCTCCTTTGTGGCAGACCA \\
\hline & lncRNA PAPSS1-R & TGCTCACTCACCCGACCCTT \\
\hline \multirow{2}{*}{12} & lncRNA ERN1-F & TTGTCCATTCCTGCCTGGGA \\
\hline & lncRNA ERN1-R & CAAGTGGTCGGCAGGAAACA \\
\hline \multirow{2}{*}{13} & $\operatorname{lncRNA}$ AP1M1-F & TACCACGCCTGGCCTATTCC \\
\hline & lncRNA AP1M1-R & AGAGGACTTCTATCTGGAACACACA \\
\hline \multirow{2}{*}{14} & lncRNA RAB27A-F & GGTGAGCATTTGGACTGGTTCC \\
\hline & lncRNA RAB27A-R & AGTGGTTCCAATTTCCССТССT \\
\hline \multirow{2}{*}{15} & lncRNA GNPTAB-F & GAAGGGGCAAGAATGGCTGC \\
\hline & lncRNA GNPTAB-R & TCTCTTCCTATGTCCTTCACATGAC \\
\hline \multirow{2}{*}{16} & GAPDH-F & AGAAGGCTGGGGCTCATTTG \\
\hline & GAPDH-R & AGGGGCCATCCACAGTCTTC \\
\hline
\end{tabular}

expression of lncRNAs RAB27A and GNPTAB was enhanced in the ACLF group with respect to the AsC group. In conclusion, we confirmed the abnormal expression of lncRNAs using RNA-Seq data by qRT-PCR experiments in a larger patient cohort. The results suggested that the aberrant expression changes in lncRNAs could play an important role in the progression of AsC to ACLF and that these lncRNAs might serve as potential biomarkers of ACLF, which has to be further studied.

3.4. Target Gene Prediction and Functional Analysis of Differentially Expressed lncRNAs. The regulation of lncRNAs can be classified into two groups: the first is cis-regulation, wherein lncRNA can regulate neighboring protein-coding genes, and the second is trans-regulation. The biological functions of differentially expressed lncRNAs in ACLF patients are not known; therefore, we predicted the targets of differentially expressed lncRNAs using previously described methods [13]. We defined the potential proteincoding gene targets as those located within the $100 \mathrm{~kb}$ flanking regions of differentially expressed lncRNAs. Then, based on these adjacent protein-coding genes, we predicted the functions of the differentially expressed lncRNAs. As shown in Figure 3, GO term and KEGG pathway enrichment indicated that the adjacent protein-coding genes were enriched for functions such as transcription and translation initiation. GO analysis revealed that the histone methylation function was significantly enriched. Zhang et al. have found that suppressor of cytokine signaling 1 gene promoter methylation is associated with acute-on-chronic hepatitis B liver failure [33]. Thus, the differentially expressed lncRNAs flanking these methylation associated protein-coding genes may also play an important role in mediating liver failure of ACLF patients. In addition, adjacent protein-coding genes were significantly enriched for the WNT signaling pathway. It is known that the WNT signaling pathway plays crucial roles 


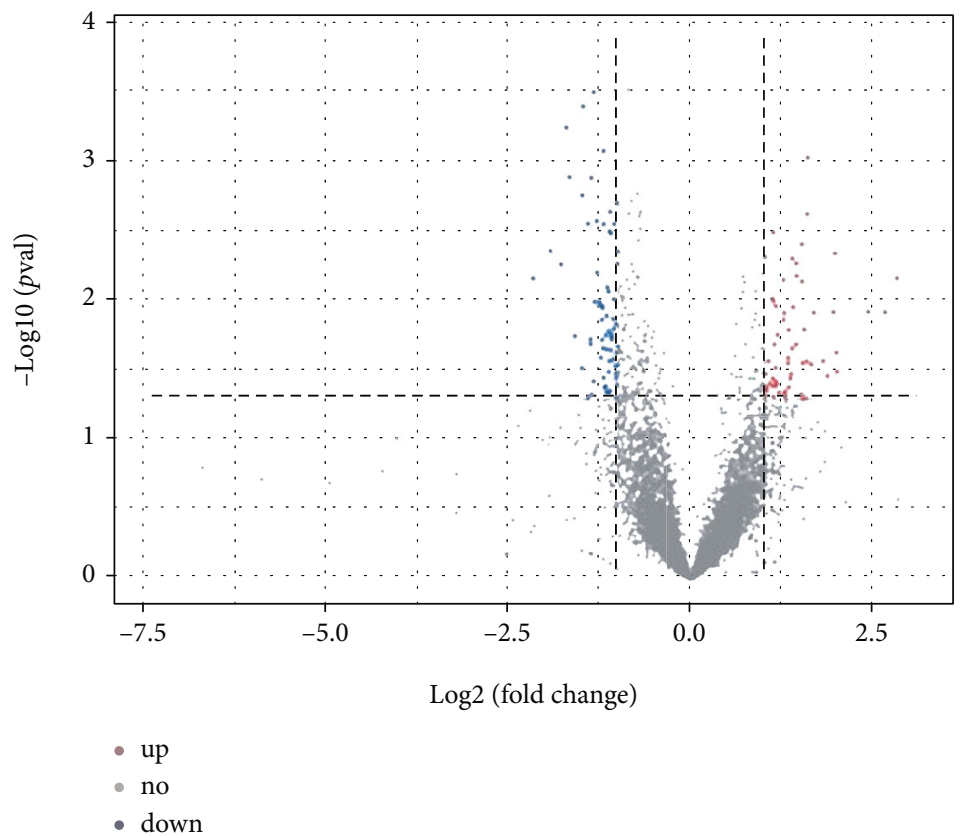

(a)

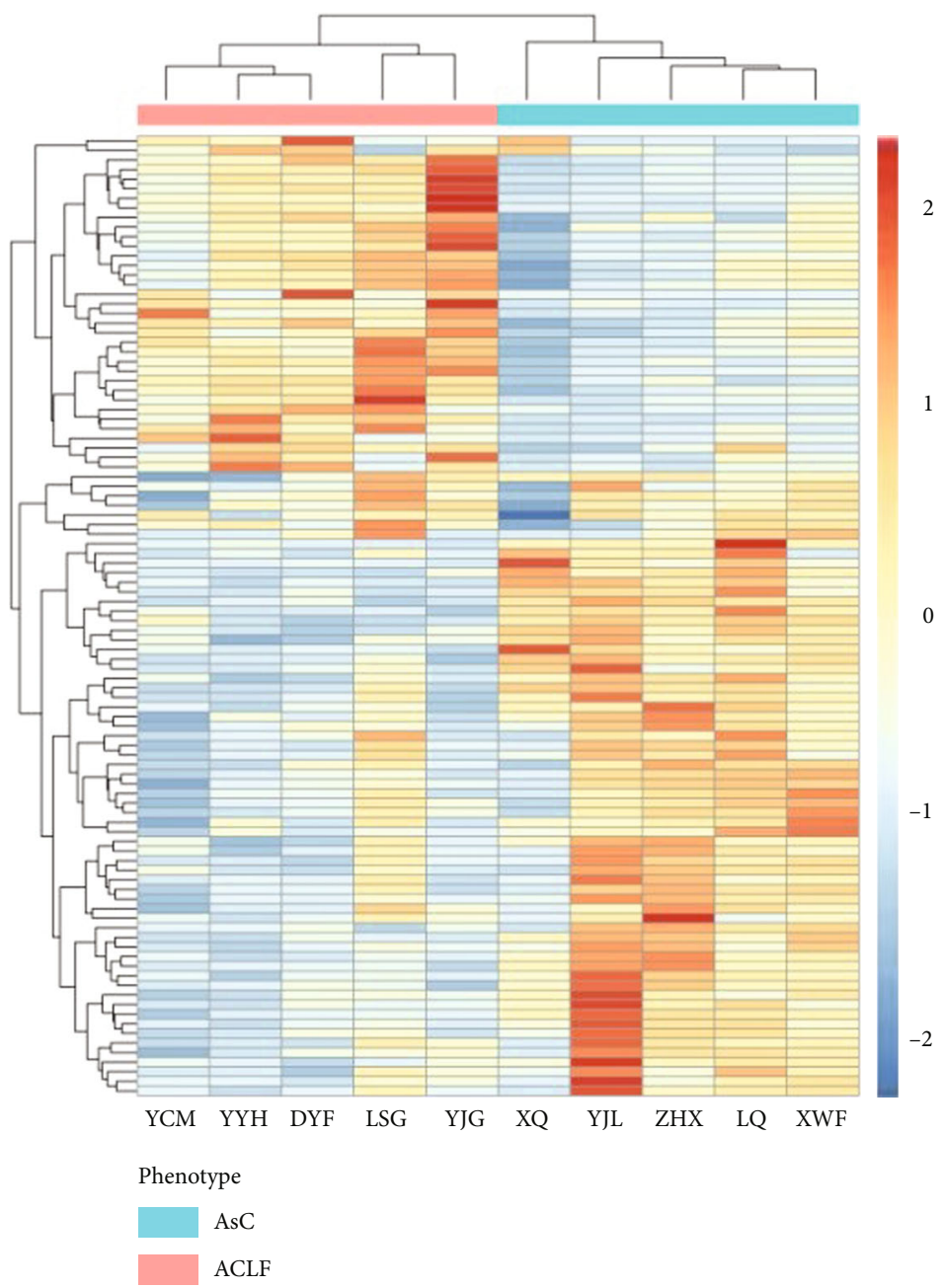

(b)

FIgUre 1: Continued. 


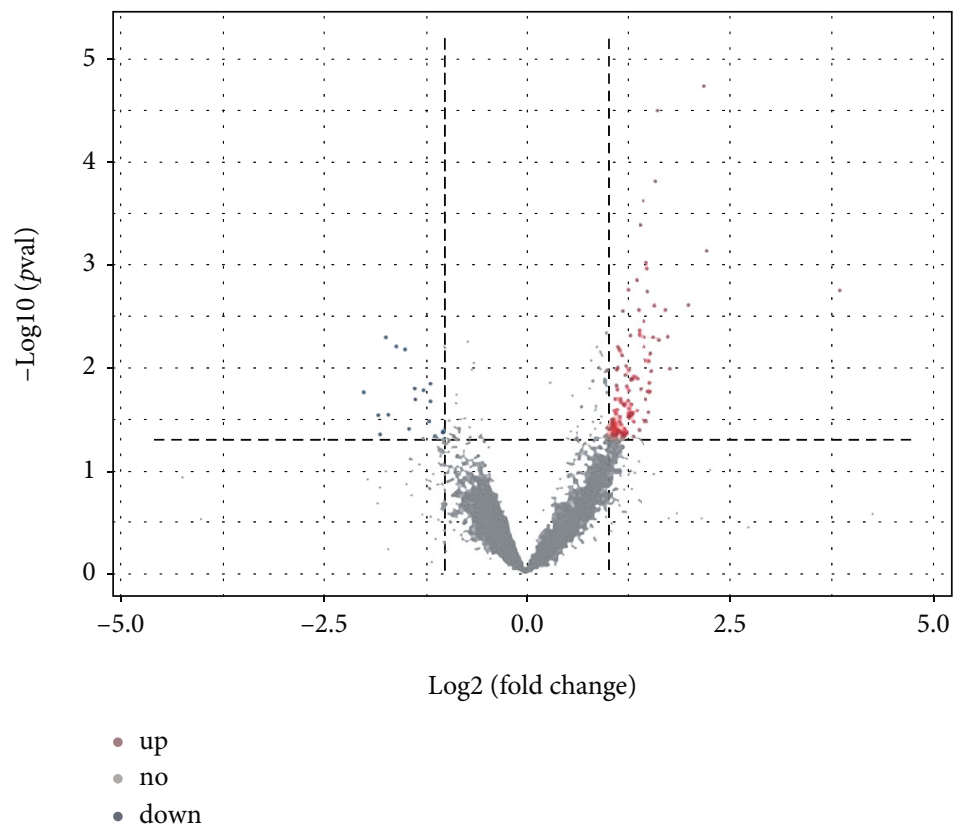

(c)

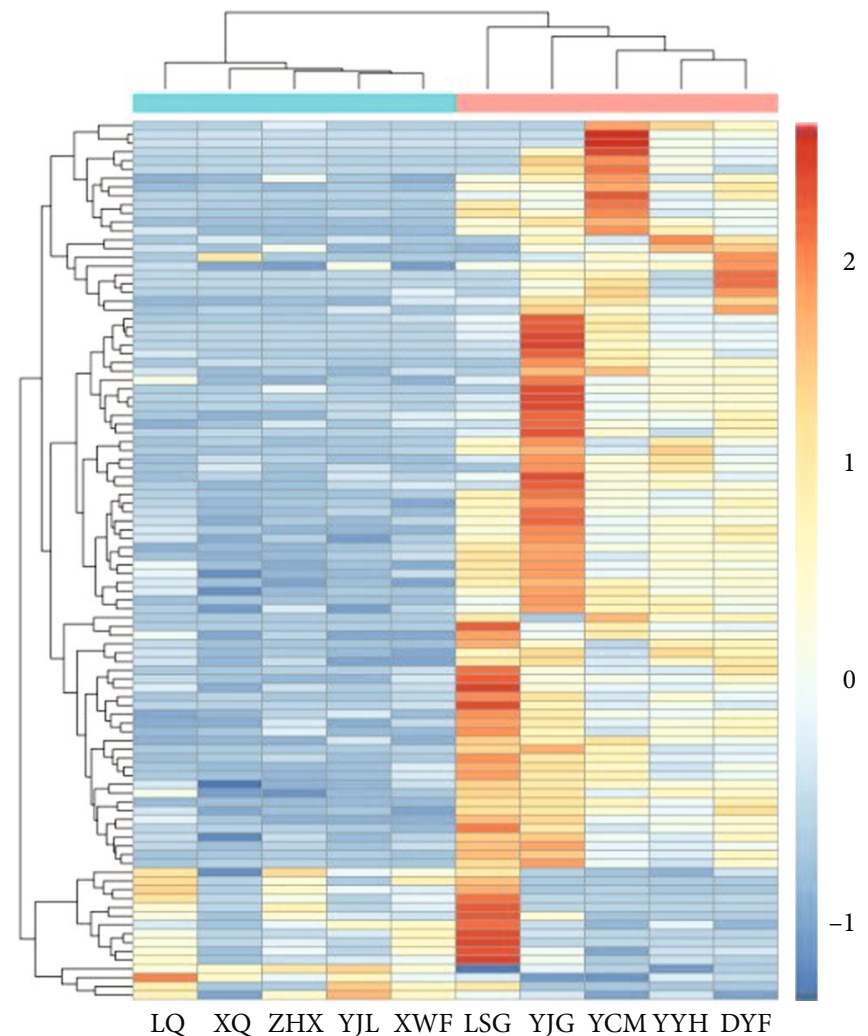

Phenotype

asC

aCLF

(d)

FIGURE 1: Differentially expressed lncRNAs in the progression of AsC to ACLF. (a) A volcano plot illustrating the fold changes and $p$ values for protein-coding genes. (b) A heat map of top 100 differentially expressed protein-coding genes. (c) A volcano plot illustrating the fold changes and $p$ values of lncRNAs. (d) A heat map of top 100 differentially expressed lncRNAs. 


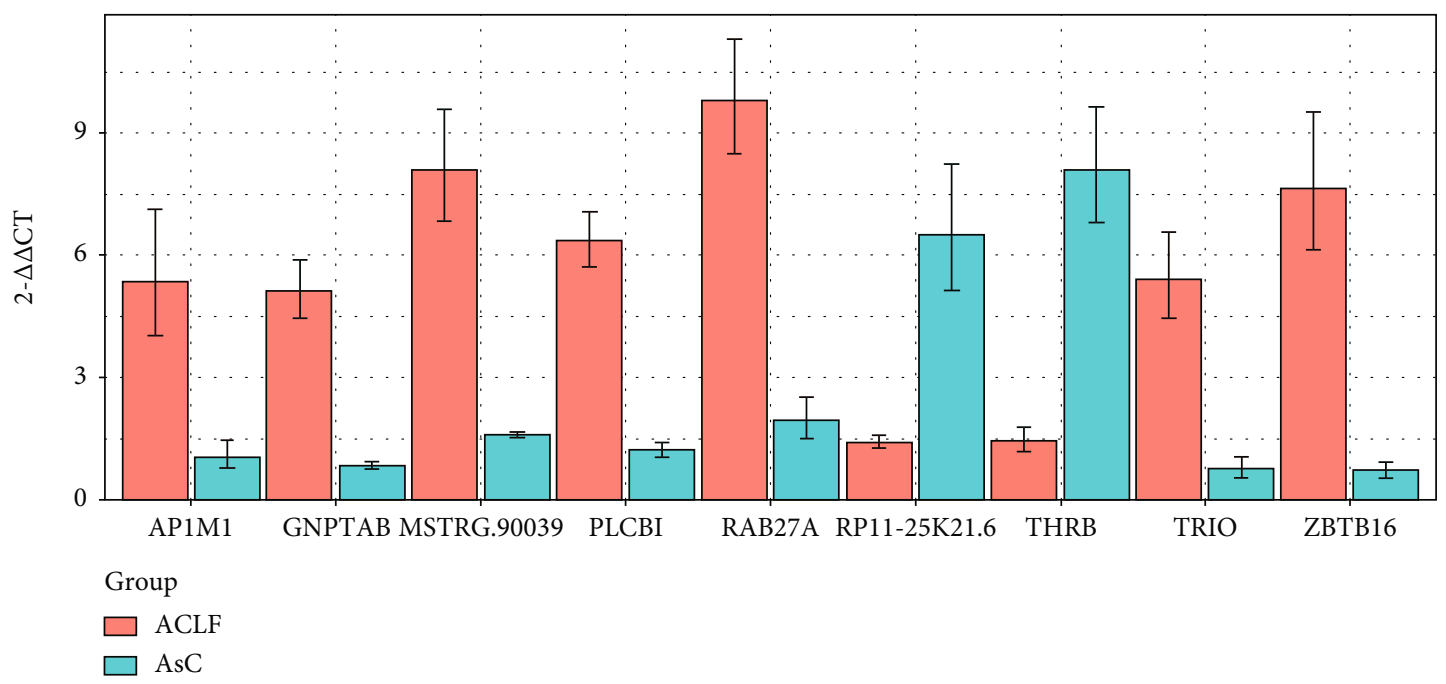

(a)

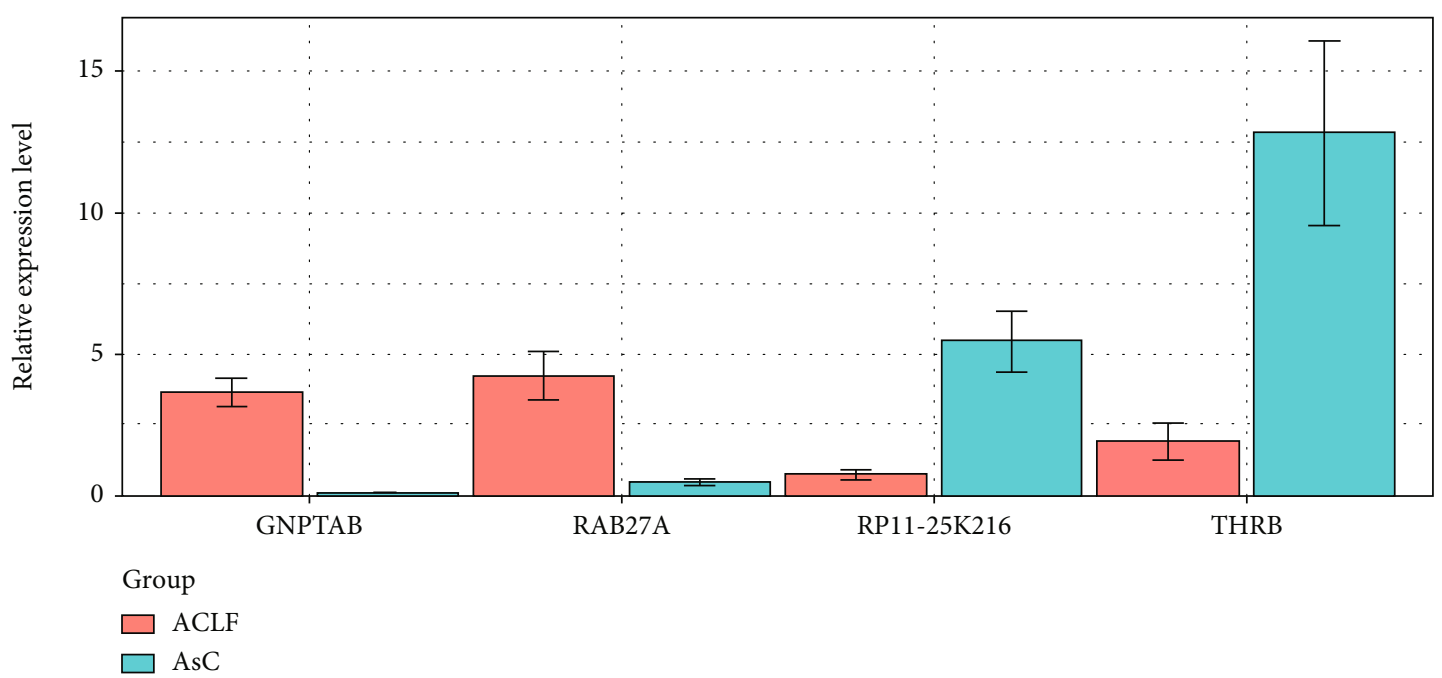

(b)

FIGURE 2: Validation of differentially expressed lncRNAs by qRT-PCR with statistical significance in the cohort comprising 5 patients with ACLF and 5 patients with hepatitis B virus (a) and another larger cohort comprising 80 patients with ACLF and 65 patients with AsC (b).

in various biological processes, such as development, proliferation, differentiation, growth, and regeneration [34, 35]. Accumulating evidences have shown that the activated WNT signaling pathway is involved in liver diseases, such as liver fibrosis, and in liver cell regeneration $[34,36]$. Our findings implied that lncRNAs may regulate adjacent protein-coding WNT genes, dysregulate the WNT signaling pathway, involved in the pathogenesis of ACLF. In total, based on the adjacent protein-coding genes of differentially expressed lncRNAs, we found that these lncRNAs may be involved in key biological functions and pathways which participate in the progression of AsC to ACLF.

A potential cis network was constructed between differentially expressed lncRNAs and protein-coding genes based on the predicted target relationships between them. As shown in Figure 4, the whole network consisted of 69 network nodes and 150 connections among 45 protein-coding genes and 24 lncRNAs. Moreover, our data showed that one mRNA might be regulated by multiple lncRNAs, and at the same time, one lncRNA may also regulate multiple protein-coding genes, suggesting complex regulatory relationships between lncRNAs and protein-coding genes.

\section{Discussion}

Chronic HBV infection is tightly associated with the occurrence of ACLF. However, the underlying pathogenesis of the progression from chronic HBV infection to ACLF is not fully understood. Accumulating evidences have shown that lncRNAs play key roles in the pathways underlying liver diseases or liver cancer [16, 19, 37-39]. For example, the activation of hepatic IncRNA H19 promotes cholestatic liver fibrosis through ZEB1-EpCAM signaling pathway in mice [15]. IncRNA HULC is specifically and highly upregulated in liver cancer, and it is associated with intrahepatic metastases, HCC recurrence, and postoperative survival $[19,20]$. We 

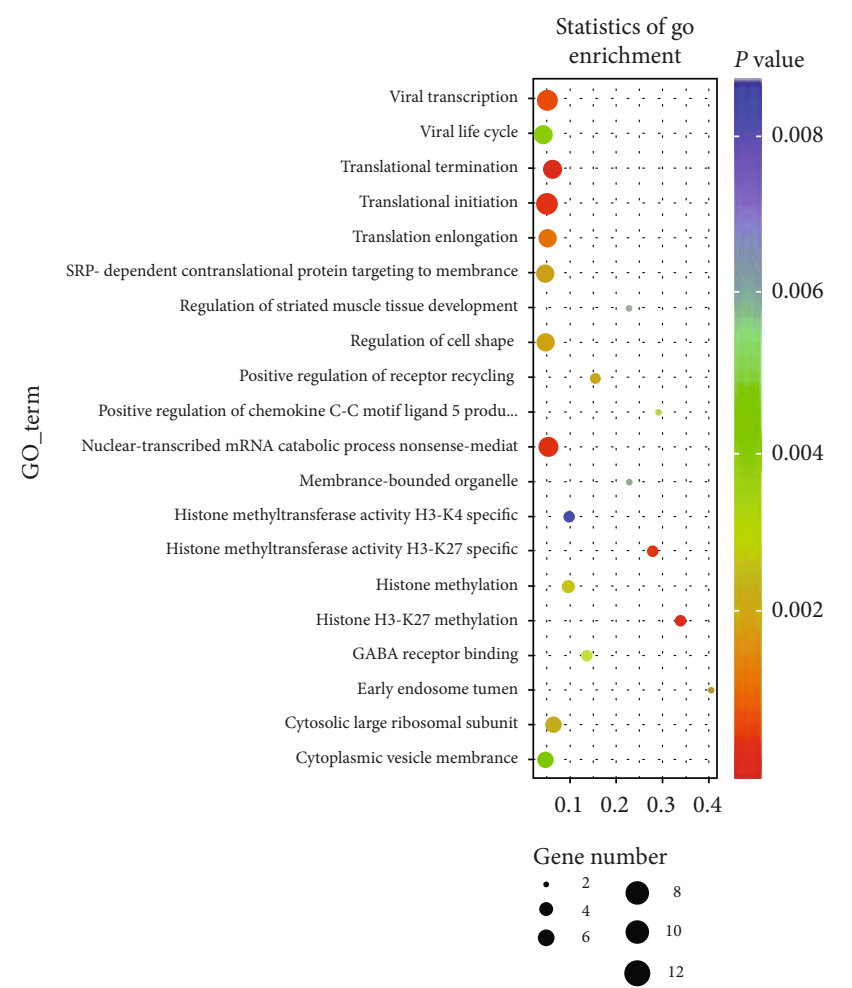

(a)

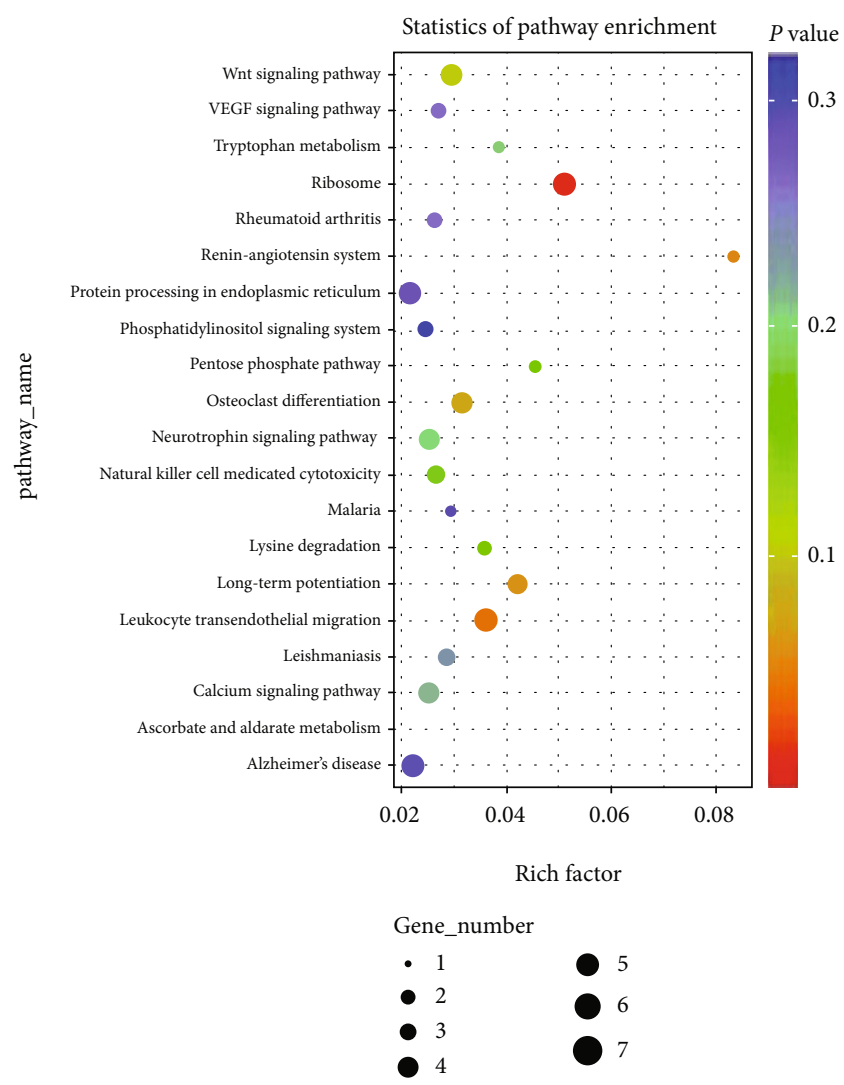

(b)

FIGURE 3: Significantly enriched GO terms (a) and KEGG pathways (b) of differentially expressed lncRNAs. GO term and KEGG pathway enrichment analyses of differentially expressed lncRNAs were performed using adjacent protein-coding genes located within the flanking $100 \mathrm{~kb}$. 


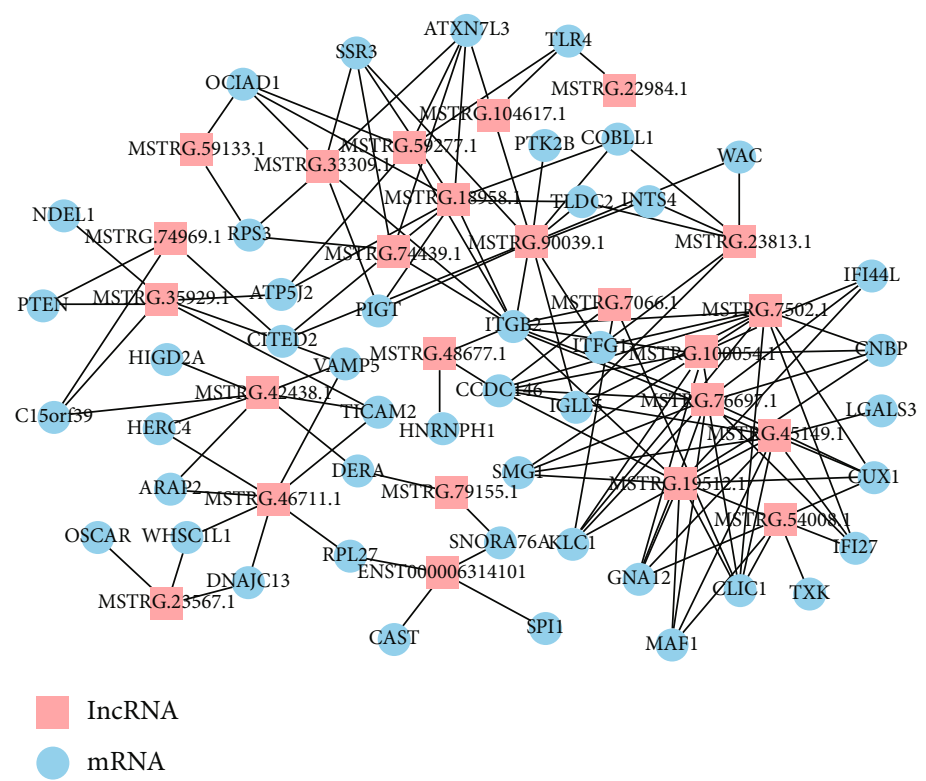

FIGURE 4: The network of lncRNAs and protein-coding genes.

wondered whether lncRNAs can be involved in the progression from chronic HBV infection to ACLF, and which lncRNA can be used as potential biomarkers to predict ACLF. Therefore, analyzing the expression profiles of lncRNAs could provide new insights for understanding the aetiology and pathophysiology of the progression from chronic HBV infection to ACLF.

In this study, we used a deep sequencing technology to characterize the expression landscape of lncRNAs in 5 ACLF and 5 chronic HBV infection patients. We analyzed differential expression of lncRNAs and predicted target proteincoding genes to identify ACLF-associated differentially expressed lncRNAs and to infer the potential functional relevance of these lncRNAs during the progression from chronic HBV infection to ACLF.

Through transcriptome analysis, we obtained 9733 lncRNAs including 406 known lncRNAs and 9327 novel lncRNAs during the progression of AsC to ACLF. Some well-known lncRNAs, such as lncRNA GAS5, MALAT1, and NEAT1, were expressed. In agreement with the results of previous studies [40,41], we observed higher variability in the number of expressed lncRNAs than in proteincoding genes. However, the exon number, transcript length, and expression levels of lncRNAs were comparable with that of protein-coding genes. The predicted ORF length of lncRNAs was also less than that of protein-coding genes, and this result was consistent with the lower coding potential of lncRNAs described in the previous research [13].

Through comparison of ACLF patients and hepatitis B virus carriers, we found 407 lncRNAs differentially expressed in ACLF, including 155 upregulated and 252 downregulated lncRNAs. On the basis of the differentially expressed lncRNAs, the ACLF patients and hepatitis B virus carriers could be divided into two distinct groups. For example, lncRNA THRB showed 3.2-fold higher expression in ACLF patients than in hepatitis $B$ virus carriers. Then, we chose
15 differentially expressed lncRNAs and performed qRTPCR experiments to further validate the expression of these lncRNAs in the two patient cohorts. Nine out of the 15 differentially expressed lncRNAs showed similar expression levels between qRT-PCR experiments and in RNA-Seq data. Four out of these 9 differentially expressed lncRNAs also exhibited significant differential expression between 80 ACLF patients and 65 hepatitis $B$ virus carriers by qRT-PCR. IncRNA RP11-25K21.6 and THRB were downregulated, and lncRNA RAB27A and GNPTAB were upregulated in ACLF patients compared with hepatitis B virus carriers. Thus, the aberrant expression of lncRNAs may be involved in the progression of AsC to ACLF. These findings could lead towards a better understanding of the function of dysregulated lncRNAs in the etiology of the progression from chronic $\mathrm{HBV}$ infection to ACLF.

Functional enrichment analysis revealed that the significantly differentially expressed lncRNAs were involved in histone methylation, the WNT signaling pathway, and other processes. In a previous study, methylation of the suppressor of cytokine signaling 1 gene promoter has been demonstrated to be associated with acute-on-chronic hepatitis B liver failure [33]. Thus, differentially expressed lncRNAs flanking these methylation-associated protein-coding genes may also play an important role in liver failure of ACLF patients. The WNT signaling pathway is involved in liver diseases and liver cell regeneration, such as liver fibrosis [34]. In our study, we found that the differentially expressed lncRNAs flanking protein-coding genes were involved in the WNT signaling pathway, implying that lncRNA may regulate adjacent protein-coding genes to induce the aberrant expression of WNT signaling pathway components, which is involved in the pathogenesis of ACLF. These findings have suggested that the differentially expressed lncRNAs may play a role in key pathways involved in the progression of AsC to ACLF. We also constructed a network of differentially expressed 
lncRNA and protein-coding gene consisting of 45 proteincoding genes and 24 lncRNAs and found that proteincoding genes may be regulated by multiple lncRNAs, and one lncRNA may also regulate multiple protein-coding genes.

Accumulation studies have reported that many lncRNAs participate in the HBV propagation. For example, lncRNA HOTAIR can regulate transcription and replication of $\mathrm{HBV}$ through promoting SP1 transcription factor [42]. IncRNA PCNAP1 promotes HBV replication and accelerates hepatocarcinogenesis via miR-154/PCNA/HBV covalently closed circular DNA signaling pathways [43]. In HBV-related hepatocellular carcinoma, lncRNA activates HBV through regulating $\mathrm{HBx} / \mathrm{STAT} 3 / \mathrm{miR}-539 / \mathrm{APOBEC} 3 \mathrm{~B}$ axis, thereby promoting the progression of $\mathrm{HBV}$-related hepatocellular carcinoma [44]. Thus, lncRNA is essential for HBV propagation. Additionally, Jiang et al. have confirmed that 132 novel lincRNAs whose expression levels are globally associated with ages [45]. Due to the small sample size, the ages of patients in our study were quite different between the AsC group and the ACLF group. Thus, the abnormal expression of lncRNAs may be related to ages of patients. This is a shortcoming in our research. The underlying connection among lncRNA, age, and ACLF still needs further research.

\section{Conclusions}

In conclusion, our study provides a novel compendium of lncRNAs expressed in the progression of chronic HBV infection to ACLF. We characterized the expression landscape of lncRNAs and identified the expression patterns of lncRNAs significantly altered in ACLF. Compared with the patients with chronic HBV infection, there were 407 lncRNAs were significantly dysregulated in ACLF patients, including 155 upregulated and 252 downregulated lncRNAs. These abnormal expressed lncRNAs may participate in the progression of ACLF. Furthermore, we validated these altered expression patterns by qRT-PCR in two patient cohorts, including RP11-25K21.6, THRB, PLCB1, TRIO, DOK6, APTX, ST3GAL4, MSTRG.7502, ZBTB16, MSTRG.90039, PAPSS1, ERN1, AP1M1, RAB27A, and GNPTAB. Aberrantly expressed lncRNAs were found to be involved in specific biological processes and related pathways that could contribute to the pathogenesis of ACLF. The results of the present study may provide a foundation for future study of the role of lncRNAs in the progression of chronic HBV infection to ACLF. Identification of dysregulated lncRNAs involved in this progression may contribute to the development of novel diagnostic biomarkers or drug targets for the treatment of ACLF. However, further research is required to determine the detailed molecular mechanisms underlying the action of significantly dysregulated lncRNAs in ACLF.

\section{Data Availability}

The data used to support the findings of this study are available from the corresponding author upon request.

\section{Conflicts of Interest}

The authors declare that there is no conflict of interest.

\section{Authors' Contributions}

Xiaoyu Fu, Da Cheng, Yi Ouyang, and Ying Li analyzed the data. Ronghua Li wrote the paper. Shifang Peng and Lei Fu designed the study.

\section{Acknowledgments}

This study was supported by the National Natural Science Foundation of China (No. 81770623, No. 81800532, No. $81974080)$ and the National Science and Technology Major Project (2017ZX10202201).

\section{Supplementary Materials}

Supplementary 1. Supplementary Figure 1: a bioinformatics computational pipeline was developed for identification of known and novel lncRNAs involved in ACLF.

Supplementary 2. Supplementary Figure 2: comparison of lncRNAs and mRNAs. (A) Expression levels of lncRNAs and mRNAs; (B) Transcript length of lncRNAs and mRNAs; (C) Exon numbers of lncRNAs and mRNAs; (D) ORFs length of lncRNAs and mRNAs.

Supplementary 3. Supplementary Figure 3: validation of differentially expressed lncRNAs by qRT-PCR without statistical significance in the patient cohort of 5 ACLF and 5 hepatitis $B$ virus carrier (A) and a new patient cohort including $80 \mathrm{ACLF}$ and $65 \mathrm{AsC}$ patients (B).

Supplementary 4. Supplementary Table 1: the RNA-Seq data and mapping rates for the 10 samples.

Supplementary 5. Supplementary Table 2: clinical characteristics of 10 patients.

Supplementary 6. Supplementary Table 3: clinical characteristics of 145 patients.

\section{References}

[1] C. Trepo, H. L. Chan, and A. Lok, "Hepatitis B virus infection," Lancet, vol. 384, no. 9959, pp. 2053-2063, 2014.

[2] the APASL ACLF Working Party, S. K. Sarin, C. K. Kedarisetty et al., "Acute-on-chronic liver failure: consensus recommendations of the Asian Pacific Association for the Study of the Liver (APASL) 2014," Hepatology International, vol. 8, no. 4, pp. 453-471, 2014.

[3] G. J. Xie, H. Y. Zhang, Q. Chen et al., "Changing etiologies and outcome of liver failure in Southwest China," Virology Journal, vol. 13, no. 1, p. 89, 2016.

[4] S. K. Asrani, D. A. Simonetto, and P. S. Kamath, "Acute-onchronic liver failure," Clinical Gastroenterology and Hepatology, vol. 13, no. 12, pp. 2128-2139, 2015.

[5] U. A. Ørom, T. Derrien, M. Beringer et al., "Long noncoding RNAs with enhancer-like function in human cells," Cell, vol. 143, no. 1, pp. 46-58, 2010. 
[6] C. P. Ponting, P. L. Oliver, and W. Reik, "Evolution and functions of long noncoding RNAs," Cell, vol. 136, no. 4, pp. 629641, 2009.

[7] P. Carninci, T. Kasukawa, S. Katayama et al., "The transcriptional landscape of the mammalian genome," Science, vol. 309, no. 5740, pp. 1559-1563, 2005.

[8] J. L. Rinn, M. Kertesz, J. K. Wang et al., "Functional Demarcation of Active and Silent Chromatin Domains in Human _HOX_Loci by Noncoding RNAs," Cell, vol. 129, no. 7, pp. 1311-1323, 2007.

[9] M. Guttman and J. L. Rinn, "Modular regulatory principles of large non-coding RNAs,” Nature, vol. 482, no. 7385, pp. 339346, 2012.

[10] M. Guttman, J. Donaghey, B. W. Carey et al., "lincRNAs act in the circuitry controlling pluripotency and differentiation," Nature, vol. 477, no. 7364, pp. 295-300, 2011.

[11] R. J. Taft, K. C. Pang, T. R. Mercer, M. Dinger, and J. S. Mattick, "Non-coding RNAs: regulators of disease," The Journal of Pathology, vol. 220, no. 2, pp. 126-139, 2010.

[12] B. K. Dey, A. C. Mueller, and A. Dutta, "Long non-coding RNAs as emerging regulators of differentiation, development, and disease," Transcription, vol. 5, no. 4, article e944014, 2014.

[13] M. N. Cabili, C. Trapnell, L. Goff et al., "Integrative annotation of human large intergenic noncoding RNAs reveals global properties and specific subclasses," Genes \& Development, vol. 25, no. 18, pp. 1915-1927, 2011.

[14] S. Augui, E. P. Nora, and E. Heard, "Regulation of Xchromosome inactivation by the X-inactivation centre," Nature Reviews. Genetics, vol. 12, no. 6, pp. 429-442, 2011.

[15] Y. Song, C. Liu, X. Liu et al., "H19 promotes cholestatic liver fibrosis by preventing ZEB1-mediated inhibition of epithelial cell adhesion molecule," Hepatology, vol. 66, no. 4, pp. 11831196, 2017.

[16] K. Zhang, X. Han, Z. Zhang et al., "The liver-enriched lncLFAR1 promotes liver fibrosis by activating TGF $\beta$ and Notch pathways," Nature Communications, vol. 8, no. 1, p. 144, 2017.

[17] C. H. Li and Y. Chen, "Targeting long non-coding RNAs in cancers: progress and prospects," The International Journal of Biochemistry \& Cell Biology, vol. 45, no. 8, pp. 1895-1910, 2013.

[18] C. Li, J. Chen, K. Zhang, B. Feng, R. Wang, and L. Chen, "Progress and prospects of long noncoding RNAs (lncRNAs) in hepatocellular carcinoma," Cellular Physiology and Biochemistry, vol. 36, no. 2, pp. 423-434, 2015.

[19] S. P. Li, H. X. Xu, Y. Yu et al., "LncRNA HULC enhances epithelial-mesenchymal transition to promote tumorigenesis and metastasis of hepatocellular carcinoma via the miR200a-3p/ZEB1 signaling pathway," Oncotarget, vol. 7, no. 27, pp. 42431-42446, 2106.

[20] J. Wang, X. Liu, H. Wu et al., "CREB up-regulates long noncoding RNA, HULC expression through interaction with microRNA-372 in liver cancer," Nucleic Acids Research, vol. 38, no. 16, pp. 5366-5383, 2010.

[21] Z. Wang, M. Gerstein, and M. Snyder, "RNA-Seq: a revolutionary tool for transcriptomics," Nature Reviews. Genetics, vol. 10, no. 1, pp. 57-63, 2009.

[22] C. Trapnell, B. A. Williams, G. Pertea et al., "Transcript assembly and quantification by RNA-Seq reveals unannotated transcripts and isoform switching during cell differentiation," Nature Biotechnology, vol. 28, no. 5, pp. 511-515, 2010.
[23] P. Martin, "Immune-tolerant hepatitis B: maybe a misnomer but still hard to treat," Hepatology, vol. 69, no. 6, pp. 23152317, 2019.

[24] S. Maimone, G. Caccamo, G. Squadrito et al., "A combination of different diagnostic tools allows identification of inactive hepatitis B virus carriers at a single time point evaluation," Liver international: official journal of the International Association for the Study of the Liver, vol. 37, no. 3, pp. 362-368, 2017.

[25] C. Trapnell, L. Pachter, and S. L. Salzberg, "TopHat: discovering splice junctions with RNA-Seq," Bioinformatics, vol. 25, no. 9, pp. 1105-1111, 2009.

[26] M. Pertea, G. M. Pertea, C. M. Antonescu, T. C. Chang, J. T. Mendell, and S. L. Salzberg, "StringTie enables improved reconstruction of a transcriptome from RNA-seq reads," Nature Biotechnology, vol. 33, no. 3, pp. 290-295, 2015.

[27] L. Kong, Y. Zhang, Z. Q. Ye et al., "CPC: assess the proteincoding potential of transcripts using sequence features and support vector machine," Nucleic Acids Research, vol. 35, no. 2, pp. W345-W349, 2007.

[28] L. Sun, H. Luo, D. Bu et al., "Utilizing sequence intrinsic composition to classify protein-coding and long non-coding transcripts," Nucleic Acids Research, vol. 41, no. 17, p. e166, 2013.

[29] C. Xie, X. Mao, J. Huang et al., "KOBAS 2.0: a web server for annotation and identification of enriched pathways and diseases," Nucleic Acids Research, vol. 39, no. 2, pp. W316W322, 2011.

[30] F. M. Gebhardt, H. A. Scott, and P. R. Dodd, "Housekeepers for accurate transcript expression analysis in Alzheimer's disease autopsy brain tissue," Alzheimers Dement, vol. 6, no. 6, pp. $465-474,2010$.

[31] A. Conesa, S. Gotz, J. M. Garcia-Gomez, J. Terol, M. Talon, and M. Robles, "Blast2GO: a universal tool for annotation, visualization and analysis in functional genomics research," Bioinformatics, vol. 21, no. 18, pp. 3674-3676, 2005.

[32] A. C. Frazee, G. Pertea, A. E. Jaffe, B. Langmead, S. L. Salzberg, and J. T. Leek, "Ballgown bridges the gap between transcriptome assembly and expression analysis," Nature Biotechnology, vol. 33, no. 3, pp. 243-246, 2015.

[33] J. J. Zhang, Y. C. Fan, Z. H. Zhang et al., "Methylation of suppressor of cytokine signalling 1 gene promoter is associated with acute-on-chronic hepatitis B liver failure," Journal of Viral Hepatitis, vol. 22, no. 3, pp. 307-317, 2015.

[34] J. N. Wang, L. Li, L. Y. Li, Q. Yan, J. Li, and T. Xu, "Emerging role and therapeutic implication of Wnt signaling pathways in liver fibrosis," Gene, vol. 674, pp. 57-69, 2018.

[35] J. N. Anastas and R. T. Moon, "WNT signalling pathways as therapeutic targets in cancer," Nature Reviews. Cancer, vol. 13, no. 1, pp. 11-26, 2013.

[36] M. J. Perugorria, P. Olaizola, I. Labiano et al., "Wnt- $\beta$-catenin signalling in liver development, health and disease," Nature Reviews. Gastroenterology \& Hepatology, vol. 16, no. 2, pp. 121-136, 2019.

[37] M. B. Zeisel and T. F. Baumert, "Translation and protein expression of lncRNAs: impact for liver disease and hepatocellular carcinoma," Hepatology, vol. 64, no. 2, pp. 671-674, 2016.

[38] Y. Yang, L. Chen, J. Gu et al., "Recurrently deregulated lncRNAs in hepatocellular carcinoma," Nature Communications, vol. 8, no. 1, article 14421, 2017.

[39] H. T. Liao, J. W. Huang, T. Lan et al., "Identification of the aberrantly expressed lncRNAs in hepatocellular carcinoma: a 
bioinformatics analysis based on RNA-sequencing," Scientific Reports, vol. 8, no. 1, p. 5395, 2018.

[40] T. Derrien, R. Johnson, G. Bussotti et al., "The GENCODE v7 catalog of human long noncoding RNAs: analysis of their gene structure, evolution, and expression," Genome Research, vol. 22, no. 9, pp. 1775-1789, 2012.

[41] M. Zhou, H. Zhao, X. Wang, J. Sun, and J. Su, “Analysis of long noncoding RNAs highlights region-specific altered expression patterns and diagnostic roles in Alzheimer's disease," Briefings in Bioinformatics, vol. 20, no. 2, pp. 598-608, 2019.

[42] F. Ren, J. H. Ren, C. L. Song et al., "LncRNA HOTAIR modulates hepatitis B virus transcription and replication by enhancing SP1 transcription factor," Clinical Science, vol. 134, no. 22, pp. 3007-3022, 2020.

[43] J. Feng, G. Yang, Y. Liu et al., "LncRNA PCNAP1 modulates hepatitis B virus replication and enhances tumor growth of liver cancer," Theranostics, vol. 9, no. 18, pp. 5227-5245, 2019.

[44] Y. Liu, J. Feng, M. Sun et al., "Long non-coding RNA HULC activates HBV by modulating HBx/STAT3/miR-539/APOBEC3B signaling in HBV-related hepatocellular carcinoma," Cancer Letters, vol. 454, pp. 158-170, 2019.

[45] S. Jiang, S. Cheng, L. Ren et al., "An expanded landscape of human long noncoding RNA," Nucleic Acids Research, vol. 47 , no. 15 , pp. $7842-7856$. 